\title{
Near-threshold absolute angle-differential cross sections for electron-impact excitation of argon and xenon
}

\author{
M. Allan \\ Department of Chemistry, University of Fribourg, Fribourg, Switzerland \\ O. Zatsarinny and K. Bartschat \\ Department of Physics and Astronomy, Drake University, Des Moines, Iowa 50311, USA
}

\begin{abstract}
Absolute angle-differential cross sections for electron-impact excitation of argon and xenon atoms to the lowest four $n p^{5}(n+1) s$ levels, and the $5 p^{5} 5 d[7 / 2]_{3}$ level in xenon, have been measured and calculated as a function of electron energy up to a few $\mathrm{eV}$ above threshold at a fixed scattering angle of $135^{\circ}$. For argon, very good agreement is observed between the experimental data and predictions from a Breit-Pauli $B$-spline $R$-matrix (BSR) method, in which nonorthogonal orbital sets are used to optimize the target description. The agreement is still satisfactory for the more complex xenon target, suggesting that predictions from the BSR model should already be sufficiently accurate for many modeling applications. Nevertheless, the remaining discrepancies indicate the need for further refinement of the theoretical model.
\end{abstract}

Absolute cross sections for inelastic electron scattering from rare-gas atoms are of great importance for gaseous discharge physics [1], but-except for helium-the theoretical description of these processes has remained a substantial challenge. Recently, however, significant progress has been made by means of a $B$-spline $R$-matrix (BSR) method [2-4]. The key feature of this method is the possibility of using nonorthogonal sets of term-dependent one-electron orbitals. This allows for an accurate target description with relatively small configuration expansions.

Since it is nearly impossible to measure experimentally all the data needed in most modeling applications, theoretical datasets for many cross sections and rate coefficients have become important ingredients in the various models. Consequently, benchmarking theoretical results against carefully produced, albeit selected, highly accurate experimental data is critical for the assessment of any computational model. Only data from a properly validated numerical approach can be used with confidence in subsequent applications.

For argon the BSR results were already successfully tested by comparing them with the experimentally measured total yield of atoms in metastable excited states [3]. Those experiments $[5,6]$ provided valuable information, in particular on the energies and widths of the resonance features. The earlier work on the resonant features and metastable production has been reviewed by Buckman and Clark [7]. The goal of the present study was to perform a further and even more stringent test, namely a comparison with cross sections that carry additional detailed information about the scattering process by being differential with respect to the scattering angle and selective with respect to the final state.

Most previous angle-differential work was carried out at several discrete impact energies, generally higher than the scope of the present work, and concentrated on the angular distributions at a fixed energy. Here we report absolute angle-differential cross sections for electron-impact excitation of argon and xenon atoms to the four lowest levels. We focus on the energy dependence of the cross sections as a function of incident electron energy for a fixed scattering angle, which reveals the contributions of the various resonant features to the excitation of the different final levels. Emphasis is placed on the energy range close to threshold, where a wealth of resonance features is found. This type of data has recently been obtained and compared to BSR calculations for $\mathrm{Ne}$ [8]. Previous absolute cross section measurements in $\mathrm{Ar}$ and Xe were nearly exclusively concerned with the angular dependence of the cross sections and exclude energies very close to threshold. Studies that include energies covered in the present work are those of Chutjian and Cartwright [9] and of Khakoo et al. [10] for argon, and of Khakoo et al. [11] for xenon. Experiments at energies only slightly higher than those covered by the present work were performed by Filipović et al. [12] for argon and by Filipović et al. [13] and Ester and Kessler [14] for xenon.

The present experimental results were obtained with a high-resolution electron scattering apparatus [15] involving two-stage hemispherical analyzers. The cross sections were recorded as a function of electron energy at the fixed scattering angle of $135^{\circ}$. The raw signal was corrected for the variations of the instrumental response with energy as described in detail recently [16].

The absolute inelastic values were determined in two steps. First, the values of the absolute elastic cross sections were determined at $15 \mathrm{eV}$ for $\mathrm{Ar}$ and at $12 \mathrm{eV}$ for $\mathrm{Xe}$ by normalization to the well-known helium results [17] using the relative flow method [18]. The resulting values of $6110 \mathrm{pm}^{2} / \mathrm{sr}$ for $\mathrm{Ar}$ and $7270 \mathrm{pm}^{2} / \mathrm{sr}$ for Xe, respectively, are believed to be accurate within about $\pm 15 \%$. The former value compares favorably to the result of $6500 \mathrm{pm}^{2} / \mathrm{sr} \mathrm{ob}-$ tained at the same energy and scattering angle by Srivastava et al. [19]. The latter value fits well between two measurements of Register et al. [20], who obtained $9240 \mathrm{pm}^{2} / \mathrm{sr}$ at $9.75 \mathrm{eV}$ and $4890 \mathrm{pm}^{2} / \mathrm{sr}$ at $14.75 \mathrm{eV}$. Linear interpolation between these two values of Register et al. yields $7280 \mathrm{pm}^{2} / \mathrm{sr}$ at $12 \mathrm{eV}$, in good agreement with the present result. Electron energy-loss spectra, including both the elas- 
tic and the inelastic peaks, were then recorded at constant incident energies of $15 \mathrm{eV}$ for $\mathrm{Ar}$ and at $12 \mathrm{eV}$ for $\mathrm{Xe}$, respectively, and subsequently corrected for the analyzer response function. Absolute values for the inelastic cross sections were determined from the elastic and inelastic signal intensities in the energy loss spectrum by normalizing to the absolute elastic values determined in the first step. Finally, excitation functions for the lowest four $n p^{5}(n+1) s$ levels in argon and xenon, and in xenon additionally for the $5 p^{5} 5 d\left[\frac{7}{2}\right]_{3}$ level, were recorded, corrected for the instrumental response function, and normalized to the absolute values at $12 \mathrm{eV}$ or $15 \mathrm{eV}$, where no sharp resonant features exist. The uncertainty of the inelastic cross sections is about $\pm 20 \%$ for energies more than $0.3 \mathrm{eV}$ above each threshold. At lower energies, the response function becomes more difficult to determine and the error bars increase gradually, reaching $\pm 50 \%$ very close to threshold.

The numerical calculations performed for the present work are based upon the semi-relativistic $B$-spline $R$-matrix approach described in Refs. [2-4]. Details of this particular method and references to earlier work can be found in these papers. As mentioned above, the key feature of this approach is to significantly improve the target description by using compact configuration-interaction expansions involving nonorthogonal sets of term-dependent one-electron orbitals.

Details of the computational model for $e$-Ar collisions can be found in Ref. [3] and will not be repeated here. For Xe, a similar model was used, regarding the construction of the target states and the close-coupling collision model. Specifically, our structure calculations included the following steps. We started by generating the $1 s$ to $5 p$ core orbitals from a Hartree-Fock calculation for $\mathrm{Xe}^{+}$. We then included the core correlation effect by single and double promotion of the $5 \mathrm{~s}$ and $5 p$ orbitals to $\overline{5} \ell$ correlated orbitals, but we only kept configurations with expansion coefficients greater than 0.05 . This left the $5 s 5 p^{5} \overline{5} d, 5 s^{2} 5 p^{3} \overline{5} d^{2}$, and $5 s^{2} 5 p^{4} \overline{5} f$ configurations. These are considered sufficient to include the most important core correlation effects with a minimum number of correlation configurations.

Then the valence orbitals were generated with the $B$-spline box-based close-coupling method described by Zatsarinny and Froese Fischer [21]. Here only the correlated $5 s^{2} 5 p^{5}$ state was used as target state in the $B$-spline boundstate expansion. In order to account for the core-valence correlation, the close-coupling expansion for the target states also included configurations with an excited core, $5 p^{4} \overline{6} \ell \overline{6} \ell^{\prime}$, where the $\overline{6} \ell(\ell=0,1,2,3)$ correlated orbitals were optimized in separate multi-configuration Hartree-Fock calculations for each term. In order to limit the bound-state expansions to an acceptable size, we only kept configurations with expansion coefficients greater than 0.005 . The above procedure allowed us to reduce the error in the binding energies for the lowest $5 p^{5} 6 s$ and $5 p^{5} 5 d$ states from $0.35 \mathrm{eV}$ to $0.13 \mathrm{eV}$, indicating that we indeed included a substantial amount of core-valence correlation.

The number of physical states that we can generate by this method depends on the radius $a$ of the $R$-matrix box. Our choice of $a=50 a_{0}$ (with $a_{0}$ denoting the Bohr radius) yields a good description for all spectroscopic states with dominant configurations $5 p^{5} 6 s, 5 p^{5} 6 p, 5 p^{5} 5 d$, and $5 p^{5} 7 s$, respectively. We emphasize that the above procedure generates nonorthogonal, term-dependent sets of radial functions for each individual state, also accounting for term mixing due to the spin-orbit interaction. In the present calculations, the atomic Hamiltonian includes all one-electron Breit-Pauli operators plus the two-electron spin-other-orbit interaction. The relativistic corrections are very important in the case of Xe, which is too heavy to expect excellent $a b$ initio results in a perturbative approach with nonrelativistic orbitals. Consequently, in order to reproduce the correct term mixing we used the experimental value of $\zeta(5 p)=1.306 \mathrm{eV}$ as the spinorbit parameter for the $5 p$ orbital. In contrast, the nonrelativistic wave function for the $5 s^{2} 5 p^{5}$ core yields $\zeta(5 p)$ $=1.075 \mathrm{eV}$.

In the subsequent scattering calculations, we included the lowest 31 physical states of $\mathrm{Xe}$ in the close-coupling expansion, namely, all states with configurations $5 p^{5} 6 s, 5 p^{5} 6 p$, $5 p^{5} 5 d$, and $5 p^{5} 7 s$, plus two pseudostates with configurations $5 p^{5} \overline{8} s$ and $5 p^{5} \overline{8} d$. The latter were optimized to account for the polarizability of the ground state to full extent. It should be noted that the target spectrum of $\mathrm{Xe}$ is significantly more complicated than that of Ar, due to the mingling of states with many different configurations. Also, since the present computational model does not include sufficient coupling to the target continuum (this would require a major increase in computational resources as well as further code development), our theoretical predictions for $e$-Xe collisions are believed to be most reliable for incident energies below $\approx 12 \mathrm{eV}$, i.e., the approximate threshold of the highest physical state included in the close-coupling expansion. For similar reasons, the accuracy of the $e$-Ar predictions might deteriorate above $\approx 15 \mathrm{eV}$.

As in the case of $e$-Ar collisions, we used the $B$-spline $R$-matrix method [4] to solve the $(N+1)$-electron collision problem. The essential idea is to expand the basis of continuum orbitals used to describe the projectile electron inside the $R$-matrix box, i.e., the region where the problem is most complicated due to the highly correlated motion of $N+1$ electrons, also in terms of a $B$-spline basis. In the present case, the box radius was again set to $50 a_{0}$ and $82 B$-splines were used for the expansion.

Finally, we used the program MJK of Grum-Grzhimailo [22] to calculate the differential cross sections from the $\boldsymbol{T}$-matrix elements produced by the asymptotic program FARM [23]. Contributions up to total electronic angular momenta $J=11$ of the projectile-target collision system were sufficient to converge the partial-wave expansion for all transitions and energies of interest.

Figure 1 exhibits the results for argon. The present experimental data agree well with those of Chutjian and Cartwright [9] at $16 \mathrm{eV}$. The original data of Khakoo et al. [10] was derived under the assumption of an elastic cross section of $8841 \mathrm{pm}^{2} / \mathrm{sr}$ at $15 \mathrm{eV}$ and $135^{\circ}$. Using our present elastic value of $6110 \mathrm{pm}^{2} / \mathrm{sr}$ for normalization would reduce their cross sections by a factor of 1.45 . Figure 1 shows these reduced values. The reduced values agree well with the present 


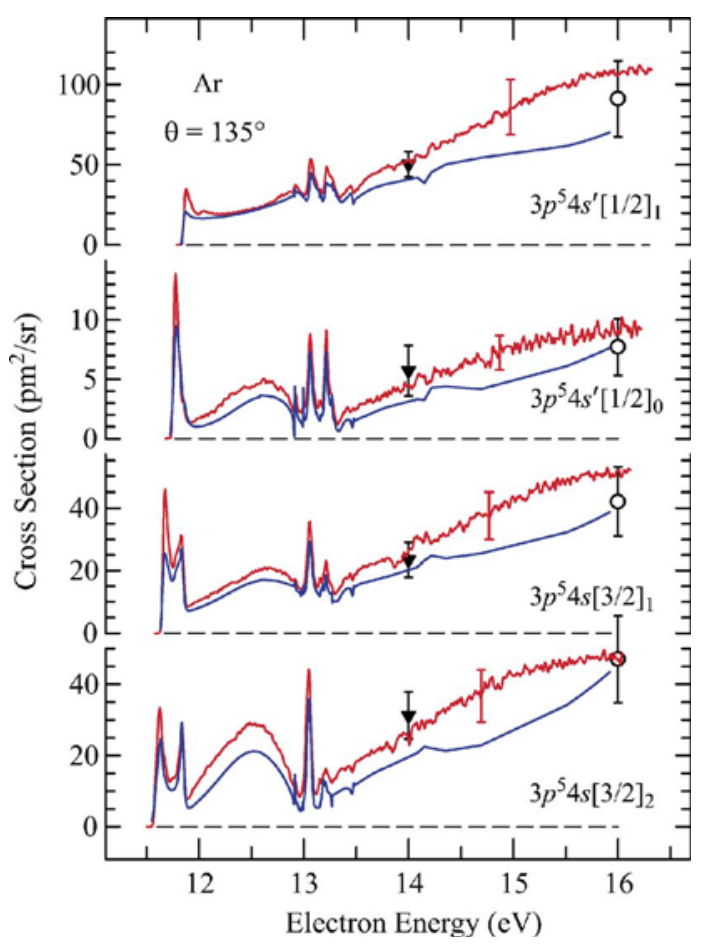

FIG. 1. (Color online) Angle-differential cross sections for electron-impact excitation of the four lowest states of argon as a function of impact energy at a fixed scattering angle of $135^{\circ}$. Ragged (red) line, present experiment; smooth (blue) line, BSR theory. Circles, Chutjian and Cartwright [9]; triangles, Khakoo et al. [10], reduced by a factor of 1.45 (see text). Representative error bars are given.

data. In this context, it is interesting to note that Yanguas-Gil et al. [24] also found an indication that the original values of Khakoo et al. [10] should be somewhat reduced. They noticed that multiplying the original cross sections of Khakoo et al. [10] by a factor of 0.5 , albeit only for the radiating states, improved the agreement of their "coherent cross section sets" (recommended for the modeling of discharge plasmas) with swarm data. Figure 1 further indicates a very satisfactory agreement between the present experiment and theory, both in terms of magnitude and the shapes and energies of the resonant features.

Figure 2 shows the corresponding results for the $5 p^{5} 6 s$ levels of xenon. At $10 \mathrm{eV}$ the data of Khakoo et al. [11] agree well with the present results for the top two spectra, but they are about two times larger for the two bottom spectra. On the other hand, the present results appear to be compatible with those of Filipović et al. [13], taken at an energy of $15 \mathrm{eV}$ (not shown in Fig. 2), although this comparison relies on smooth extrapolation of the present data to $15 \mathrm{eV}$.

Finally, Fig. 3 shows the results for excitation of the $5 p^{5}\left({ }^{2} P_{3 / 2}^{0}\right) 5 d[7 / 2]_{3}^{0}$ level of xenon. Extrapolation of our data suggests a slightly lower value than that measured at $15 \mathrm{eV}$ by Filipović et al. [13], but the difference, about $25 \%$, is within the combined error limits. The agreement between theory and experiment is reasonable for incident energies up to $\approx 12 \mathrm{eV}$. As mentioned above, the lack of coupling to the target continuum is most likely responsible for the rapid increase of the theoretical results above the experimental data for higher energies.

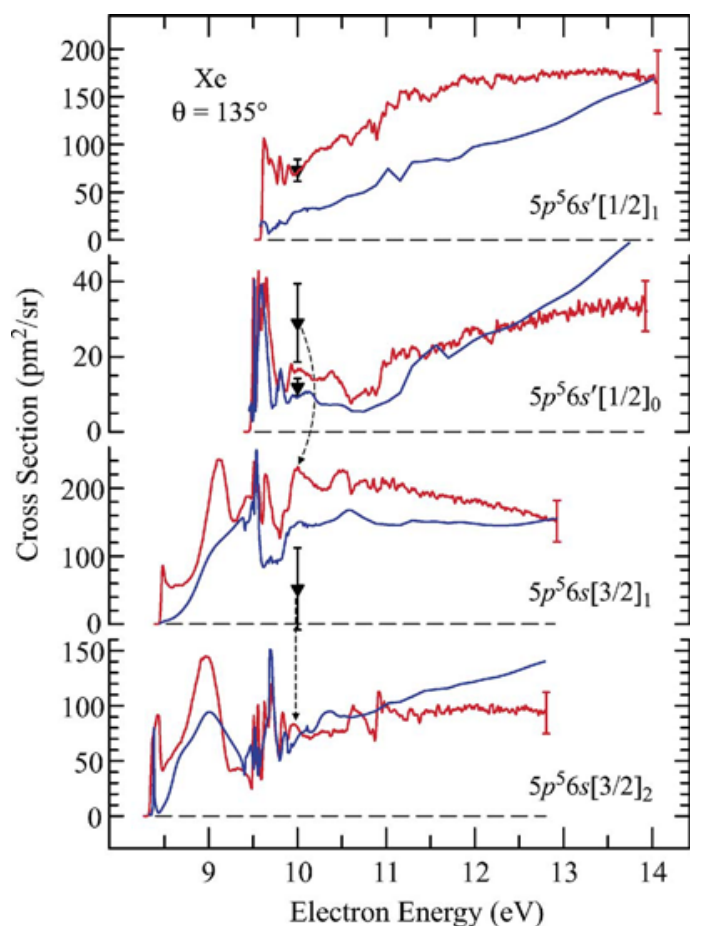

FIG. 2. (Color online) Same as Fig. 1, but for the four lowest states of xenon. Triangles, Khakoo et al. [11].

In conclusion, we have presented benchmark data for absolute angle-differential cross sections for electron-impact excitation of argon and xenon atoms. Comparison with the few existing previous data in the literature indicates good agreement with the $e$-Ar results of Chutjian and Cartwright [9] and the $e$-Xe measurements of Filipović et al. [13]. Good agreement is also found with renormalized data of Khakoo et al. [10] for Ar. Comparison for Xe $[10,11]$ revealed agreement for the upper two, but a significant difference for the lower two of the four levels considered.

For a fixed scattering angle of $135^{\circ}$, the energy dependence of the cross section is dominated by a complex resonance structure. For argon, very good agreement is observed between the experimental data and predictions from a BreitPauli $B$-spline $R$-matrix method using nonorthogonal orbital sets in the target description. The agreement is still satisfactory for the more complex xenon target, although discrepan-

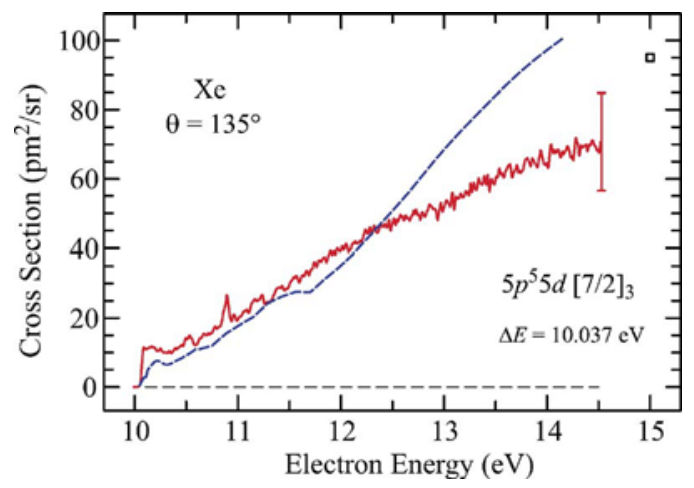

FIG. 3. (Color online) Same as Fig. 2, but for the $5 p^{5} 5 d[7 / 2]_{3}$ level of xenon. Square at $15 \mathrm{eV}$, Filipović et al. [13]. 
cies remain in details. While this indicates the need for further refinement of the theoretical model, the overall agreement between theory and experiment suggests that predictions from the BSR model should already be sufficiently accurate for many modeling applications.
One of the authors (M. A.) acknowledges support by the Swiss National Science Foundation and two of the authors (O. Z. and K. B.) acknowledge support by the United States National Science Foundation. The authors thank Professor M. A. Khakoo for comments and updated cross sections.
[1] L. G. Christophorou and J. K. Olthoff, Adv. At., Mol., Opt. Phys. 44, 59 (2000).

[2] O. Zatsarinny and K. Bartschat, J. Phys. B 37, 2173 (2004).

[3] O. Zatsarinny and K. Bartschat, J. Phys. B 37, 4693 (2004).

[4] O. Zatsarinny, Comput. Phys. Commun. 174, 273 (2006).

[5] S. J. Buckman, P. Hammond, G. C. King, and F. H. Read, J. Phys. B 16, 4219 (1983).

[6] J. M. Phillips, J. Phys. B 15, 259 (1982).

[7] S. J. Buckman and C. W. Clark, Rev. Mod. Phys. 66, 539 (1994).

[8] M. Allan, K. Franz, H. Hotop, O. Zatsarinny, and K. Bartschat, J. Phys. B 39, L139 (2006).

[9] A. Chutjian and D. C. Cartwright, Phys. Rev. A 23, 2178 (1981).

[10] M. A. Khakoo, P. Vanderventer, J. G. Childers, I. Kanik, C. J. Fontes, K. Bartschat, V. Zeman, D. H. Madison, S. Saxena, R. Srivastava, and A. D. Stauffer, J. Phys. B 37, 247 (2004).

[11] M. A. Khakoo, S. Trajmar, L. R. LeClair, I. Kanik, G. Csanak, and C. J. Fontes, J. Phys. B 29, 3455 (1996).

[12] D. M. Filipović, B. P. Marinković, V. Pejčev, and L. Vušković, J. Phys. B 33, 677 (2000).
[13] D. Filipović, B. Marinković, V. Pejčev, and L. Vušković, Phys. Rev. A 37, 356 (1988).

[14] T. Ester and J. Kessler, J. Phys. B 27, 4295 (1994).

[15] M. Allan, J. Phys. B 25, 1559 (1992).

[16] M. Allan, J. Phys. B 38, 3655 (2005).

[17] R. K. Nesbet, Phys. Rev. A 20, 58 (1979).

[18] J. C. Nickel, C. Mott, I. Kanik, and D. C. McCollum, J. Phys. B 21, 1867 (1988).

[19] S. K. Srivastava, H. Tanaka, A. Chutjian, and S. Trajmar, Phys. Rev. A 23, 2156 (1981).

[20] D. F. Register, L. Vušković, and S. Trajmar, J. Phys. B 19, 1685 (1986).

[21] O. Zatsarinny and C. Froese Fischer, J. Phys. B 35, 4669 (2002).

[22] A. N. Grum-Grzhimailo, Comput. Phys. Commun. 152, 101 (2003).

[23] V. M. Burke and C. J. Noble, Comput. Phys. Commun. 85, 471 (1995).

[24] A. Yanguas-Gil, J. Cotrino, and L. L. Alves, J. Phys. D 38, 1588 (2005). 